\title{
Secure Remote Reconfiguration of an FPGA-based Embedded System
}

\author{
An Braeken *, Jan Genoe ${ }^{\dagger}$, Serge Kubera*, Nele Mentens ${ }^{\dagger \ddagger}$, Abdellah Touhafi*, Ingrid Verbauwhede ${ }^{\ddagger}$, \\ Yannick Verbelen*, Jo Vliegen ${ }^{\dagger \ddagger}$, Karel Wouters ${ }^{\ddagger}$ \\ *Erasmushogeschool Brussel, Dept. IWT; Nijverheidskaai 170, Brussel, Belgium \\ ${ }^{\dagger}$ Katholieke Hogeschool Limburg; Agoralaan B, Diepenbeek, Belgium \\ ${ }^{\ddagger}$ Katholieke Universiteit Leuven, ESAT, SCD/COSIC; Kasteelpark Arenberg 10, Leuven, Belgium \\ Email: firstname.lastname@ \{ehb.be $\}\{$ khlim.be $\}\{$ esat.kuleuven.be $\}$
}

\begin{abstract}
This paper describes the protocol, architecture, and implementation details of an FPGA-based embedded system that is able to remotely reconfigure the FPGA, using a TCP/IP connection, in a secure way. When considering the security aspects, we imply data confidentiality, explicit key authentication and data origin authentication. Since these aspects are overhead for the main application, the system is to be as small as possible. Therefore we have focused on compactness rather than on speed for the implementation. The implemented solution exists out of 2 components: a communication part and a cryptographic part. The system can be easily integrated at any point in the design of an FPGA-based embedded system, due to the simple and modular architecture.
\end{abstract}

\section{INTRODUCTION}

Remote reconfiguration of an embedded system allows for important features such as remote status checking, remote problem solving, and the remote upgradeability of the software and reconfigurable hardware. Due to the fact that FPGAs (Field Programmable Gate Arrays) become more and more popular in a wide range of applications, we have restricted ourselves to a solution of remote reconfiguration for FPGAbased embedded systems. In this paper, we call this the STRES (Secure Techniques for Remote reconfiguration of Embedded Systems) system. In its design, STRES follows three main principles for a remote configuration tool: security, simplicity, and compactness.

- Most integrated FPGA solutions are restricted to reconfiguring the device, after the decryption of an encrypted bitstream $^{1}$, which provides data confidentiality. Authentication of the sending entity or the received data, however, can not be guaranteed. This is a huge concern since no authentication (neither on entity nor data) enables for example a man-in-the-middle attack, which can lead to the acceptation of corrupted bitstreams. Also the symmetric key, used for decryption, has to be available on the device. STRES is based on a standard protocol that offers both mutual entity authentication and mutual explicit key authentication, on top of data confidentiality.

- Many existing systems are initially developed without taking security or remote reconfiguration into account.

\footnotetext{
${ }^{1}$ a bitstream is the datastream that describes the configuration of the FPGA and hence defines its operational behavior
}

STRES can be easily integrated at any point in the design. At the embedded system side there are mainly two blocks: one communication component for communicating over TCP/IP, and one hardware IP core ${ }^{2}$ for encrypting and decrypting the communication (with negotiated keys) on the one hand and for authenticating the entities and the data on the other hand.

- For the majority of products, the cryptographic functionality is not part of the core tasks. Hence, it is desirable to use a minimum of the reconfigurable fabric for the STRES implementation. The more resources that are used by the STRES system, the less resources are available for the actual application. Compactness of the two components is hence preferred over computational performance in the STRES system.

To summarize, the main novelty of the paper is the realization of a flexible implementation for remote reconfiguration of an FPGA where communication is through TCP/IP and security based on public key cryptography in order to ensure confidentiality and authentication. Moreover, both the communication and the security component have the smallest implementation size, compared to other work in literature.

This paper is organized as follows. Section 2 describes the most relevant related work. The setting and assumptions are discussed in Section 3. Section 4 explains the cryptographic protocol and algorithms. Section 5 deals with the architecture and the implementation of the STRES system at the hardware side (FPGA). Timings and sizes of the different steps in the implementation are presented in Section 6. We conclude in Section 7 with possible future work.

\section{RELATED WORK}

Although the concept of security for remote reconfiguration is very important, there has not been a lot of research on this topic. In particular, work where both confidentiality and authentication are taken into account, is very rare. We now give an overview of the most important papers that have been published in this area.

Badrignans [2] was one of the first to consider authentication (besides confidentiality) for remote reconfiguration. How-

\footnotetext{
${ }^{2}$ an Intellectual Property (IP) core is a reusable unit of logic
} 
ever, his protocol only takes authentication of the bitstream into account. In [17] Devic et al. present a protocol which adds protection against replay attacks to data confidentiality. Their work is based on symmetric key cryptography, where the long term key is stored on the device in Non-VolatileMemory. They also do not have protection for side channel analysis in their scope. In [6], Drimer describes, amongst other things, a new and very efficient protocol for confidentiality and authentication of bitstream and entity. The main difference with our solution is that we use a standardized security protocol which has been in use for some time and still remains unbroken. Our architecture also allows an easy expansion of the cryptographic functionality. Moreover, we implemented our approach in a working prototype that we describe in this paper. As far as we are aware, we only found two papers [17] and [6] that also describe a secure protocol for remote reconfiguration together with their implementation. However the remote communication passes on RS232 in [17] and on Ethernet in [6] and both protocols are based on symmetric key cryptography. In our paper, we describe the implementation of the communication by means of TCP/IP and we implemented a dedicated crypto co-processor in order to handle the different steps of the security protocol, which are based on public key cryptography.

\section{Setting And ASSUmptions}

To provide the embedded system (ES) with facilities for inthe-field upgrading and updating, it should be able to communicate with other entities. We can distinguish three entities in this process: the ES in the field, the central reconfiguration unit (CRU), and the end user that uses or operates the ES. We make the assumption that only the CRU is managed by a trusted entity and therefore is the only entity authorized to update the ES. In this context, we assume that the CRU does not have to interact with the end user first. With three entities present there are six possibile communication channels: between every two entities there are two directions of communication. The following assumptions, regarding these channels, have been made:

- the ES will only answer to the trusted CRU, and will never initiate communications;

- the ES offers some very basic status report to the end user by means of an error log, LED codes, ...;

- the end user is not allowed to alter the configuration that runs on the ES, but can ask the CRU to do so;

- the CRU is the only entity that can initiate communication with the ES;

In the scope of this paper we will restrict ourselves to the communication channel between CRU and ES, since this the channel used for the secure, remote reconfiguration of the ES.

Another assumption, made for efficiency and security reasons, is the fact that the CRU does not need to keep track of a list of secret keys, counters, or other security related material for each ES. This would be a tempting single point of attack. Instead, it only needs a list of identification codes (IDs) and IP addresses of each ES.

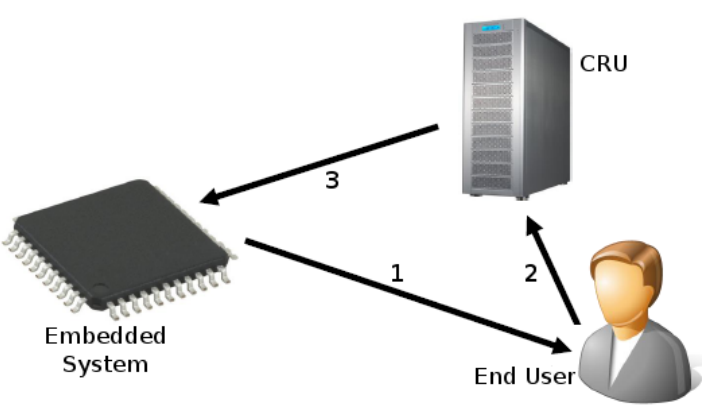

Fig. 1. Entity communication channels

\section{CRYPtOGRAPHIC PROTOCOL AND ALGORITHMS}

As mentioned above, the main goal of STRES is to use a standard protocol. This will enlarge the chances of developing a secure system for remote reconfiguration. We distinguish two different types of protocols: those based on public key cryptography and those based on symmetric key cryptography. We now shortly describe both types of protocols. Then the specific protocol for STRES is explained in more detail. Finally, we mention the cryptographic algorithms that are used in the STRES protocol.

\section{A. Public key based cryptographic protocols}

In pubic key cryptography, the most popular standard protocol is the three-phase station-to-station protocol [7]. In the protocol a shared secret key is agreed on, based on mutual entity authentication and mutual explicit key authentication. If the public keys of the communication parties are not known in advance, a certificate is sent in the second and third phase of the protocol. This slightly modified version is called full STS. Note that a certificate can be seen as the signature of one entity on the public key of the other entity. After the agreement of a shared secret session key, a symmetric cipher can be used to communicate.

The Elliptic Curve Integrated Encryption Scheme (ECIES) protocol is another protocol based on public key cryptography, which is able to send a message in an authenticated and encrypted way, together with obtaining entity authentication [1] in one-phase. This protocol can be seen as the STS protocol, where the random input of the ES is fixed to its public key, and thus reducing the number of steps in the protocol from three to one. The main danger in this protocol is the replay attack that can downgrade instead of upgrade the ES.

\section{B. Symmetric key based cryptographic protocols}

A very popular protocol based on symmetric key cryptography is the point-to-point protocol ( $\mathrm{P} 2 \mathrm{P})$ [7]. P2P tries to agree on a shared secret key, in an authenticated way. It is a threephase protocol that results in a secret shared session key that can be used for later authenticated encryption. In fact, it can be seen as the symmetric key variant of STS. Note that this protocol assumes that both parties possess an initial shared secret key. There are some simple solutions for deriving this 
initial shared secret key in order to avoid keeping track of a list of keys at the CRU. For instance, the key can be defined as the encryption of the unique device code of the embedded system under a secret master key. Another possibility is to take the signature on the unique device code of the embedded system by the CRU. For both key derivations, the final protocol slightly differs from the $\mathrm{P} 2 \mathrm{P}$ protocol in the sense that during the first phase, also the unique ID code of the ES should also be transmitted.

\section{STRES}

The STRES system is based on the full STS protocol. We have opted for this protocol since it provides the requirements we need. It enables for data confidentiality, (mutual) entity authentication and (mutual and explicit) key authentication. The STS protocol is widely used and very popular in industrial applications. Moreover, once we have an implementation based on full STS, changing to P2P or ECIES is straightforward. The choice of using the full STS protocol derives from the assumption that we do not want to store sensitive data on the CRU. Using a public key based protocol would result in a long term key for every ES issued. This requires the CRU to store every symmetric key, which is not desired in the STRES system.

Fig. 2 shows the protocol applied in STRES. For the public key part of the protocol, elliptic curve operations are used. The first use of these was reported by Koblitz and Miller in [8], [10]. We have chosen for elliptic curve cryptography since it offers the same level of security with smaller key lengths, compared to other public key cryptographic schemes.

A key pair is denoted by the tuple (private key, public key). We use a small case letter for the private key and the according capital case letter for the public key. $S_{k}$ represents a signature algorithm, HASH a hashing algorithm, $E_{k}$ an encryption algorithm, and $D_{k}$ the corresponding decryption algorithm by means of key $k$. The concatenation of messages is denoted by II.

In this implementation the protocol shown in Fig. 2 results in:

- a 256 bit session key $K$, which can be split in two halves: $k e y_{1}$ and $k e y_{2}$. The first half is used for the symmetric key encryption while the second half is used for generating the message authentication code (MAC);

- mutual, explicit key authentication;

- mutual entity authentication.

\section{Cryptographic algorithms}

If we consider the full STS protocol, the following seven functionalities at the ES side (FPGA) need to be implemented: verifying a signature (1), generating a signature (2), executing a scalar multiplication (3), encrypting a message using a symmetric key (4), decrypting a message using a symmetric key (5), calculating a hash value (6), and generating a MAC (7). The first three functionalities (1-3) can be achieved by means of a specified set of elliptic curve operations, hash function operations, and random number generations. The

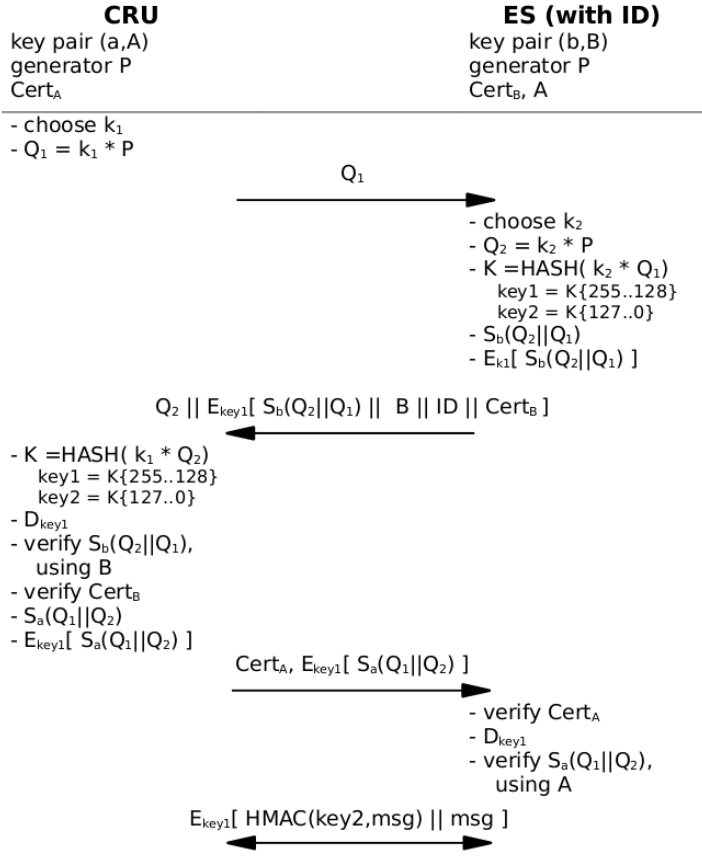

Fig. 2. STRES protocol

second two operations $(4,5)$ require a symmetric block cipher. The last two operations $(6,7)$ can both be done by a single HASH implementation using HMAC [3].

We have chosen ECDSA for the signature algorithm, SHA256 for the HASH function, AES with key length 128 bit for the symmetric key block cipher, and a true random number generator based on combining parallel oscillating circuits. The curve selected for the STRES cryptographic protocol is P-256 with prime $p=2^{256}-2^{224}+2^{192}+2^{96}-1$, which is a recommended curve by NIST [11].

When looking at Fig. 2 it shows that the public keypair for the embedded systems has to be stored on the FPGA, together with a certficate and the public key of the CRU. These values are stored in the 'Shared Memory' block of the cryptographic core (shown in Fig. 4) and hence reside in the bitstream that configures the FPGA.

\section{ARCHITECTURE AND IMPLEMENTATION}

With respect to the implementation, we restrict ourselves to the hardware side, being the FPGA-based ES. Fig. 3 shows the architecture of the FPGA-based ES, which was implemented on a Xilinx ML507 board. The FPGA is divided into a dynamic and a static zone. The dynamic region contains an application that should be securely and remotely reconfigured by means of STRES. The static zone contains the STRES system which enables secure communication and reconfiguration. Such partitioning enables a straightforward plug-in of the STRES system in any other FPGA design. The STRES system itself is also subdivided into 2 main modules: the communication core, and the cryptographic core. The former handles all the communication and reconfiguration, while the 
latter handles the cryptographic operations. Both are explained below.

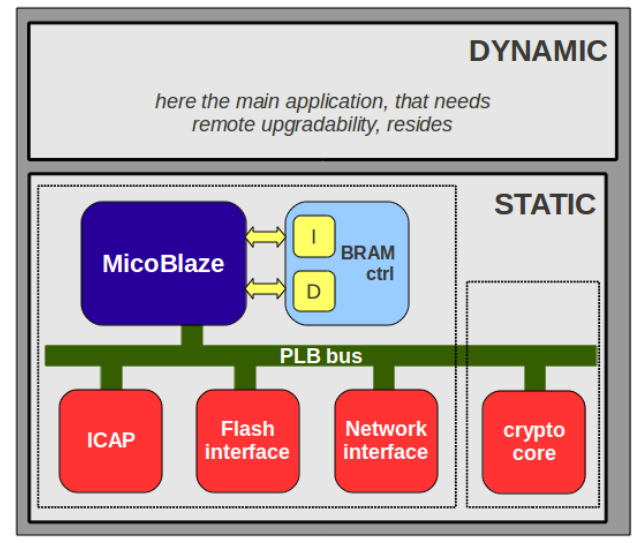

Fig. 3. Hardware architecture of FPGA-based ES

\section{A. The communication core}

As stated above the communication core handles: the communication with the CRU, and the reconfiguration of the FPGA. Since both of these tasks require some subcomponents, the communication core works on a 32 bits MicroBlaze softcore processor. Using this processor it is possible to use several IP cores which are connected to the MicroBlaze: the Internal Configuration Access Port (ICAP), the Flash interface, and the network interface. The Block RAM (BRAM) of the FPGA is used to store the data and instruction memory which is used by the MicroBlaze.

The communication protocol between the CRU and the ES gets the incoming data by using the network interface IP core. We operate in different sessions, where we first connect to a global port and ask for a private port for the transactions of the security protocol. On the second port the complete security protocol, as shown in Fig. 2, will be executed. When the new bitstream is received, it gets decrypted and its data authentication is checked. To perform these cryptographic operations the crypto core is used. When all requirements are met the bitstream will be stored in the Flash memory by using the Flash interface. There are four types of Flash: Bytewide Peripherical Interface (BPI), Serial Peripherical Interface (SPI), Platform Flash, and compact Flash. Platform Flash is programmed by means of JTAG only. Compact Flash is more complex to use in combination with the ICAP and also needs extra logic. Therefore, we only focused on BPI and SPI. From this Flash memory we will attempt to reconfigure the FPGA with the new bitstream, by using the ICAP IP core.

Continuity may be endangered if a newly received bitstream does not seem to successfully reconfigure the FPGA. Herefor a golden image is stored in the Flash memory as well. This golden image is a bitstream that only configures the static part of the FPGA, leaving the dynamic part blank. Another use of this golden image is that this is the bitstream that is used to configure the FPGA when the power comes up.
This configuation then handles a TCP/IP communication, we implemented in a small program, called nIP.

1) nIP component: The nIP component is a small software program, running on the MicroBlaze, that is able to handle TCP/IP packages. In fact, there already exist two standalone options for using TCP/IP in embedded systems: micro IP ( $\mu$ IP) [14] and light weight IP (LWIP) [15]. $\mu$ IP is the smallest one, but still requires a lot of work to port it to MicroBlaze. LWIP already exist for Xilinx FPGA, but its large size interferes with our aim for a small implementation. One could use a boot loader and place the application in external RAM. However, this would imply an extra insecure channel. Consequently, in order to limit the size of the implementation and to have maximum control over the communication, we wrote our own software, called nano IP (nIP), that contains only the strictly necessary procedures for executing the protocol shown in Fig. 2. nIP consists of the following functions and properties:

- Automatically reply on ARP requests;

- Handle large files and single packets (tested with $50 \mathrm{MB}$ );

- Create TCP packages with a single command;

- Decode a package into usable info and data;

- Handle and trigger resets (RST);

It is interesting to mention that though TCP/IP is a standard protocol, it is not always implemented in the same way. The server software, running on the CRU, is written in $C \#$. The TCP/IP protocol, as implemented in $C \#$, is as follows:

- The client sends a synchronization package to the server (SYN).

- (SYN) gets accepted by the server and replies with an acknowledge (SYN ACK).

- The client acknowledges it too (ACK) and the data transfer can be initiated by server or client.

- After the last transmitted byte, a push command is sent (PSH). In this way, the sender emphasizes that all bytes are sent and the receiver can consequently empty its receiver buffer (PSH).

- The communication will be terminated, mainly triggered by (FIN) and acknowledged by reply (FIN ACK).

\section{B. The cryptographic core}

As mentioned in Sect. IV-D, there are four primitives that need to be implemented in order to be able to execute the full STS protocol: the elliptic curve operations, a hash function, a random number generator, and a block cipher. In the STRES system four primitives are interconnected with a shared-memory scheme, which is used to provide and consume data from the primitives. Some overhead is required to make everything work together. Fig. 4 shows the general architecture of the cryptographic core.

Note that all the cryptographic primitives are constructed with compactness in mind. All of the primitives have the shared-memory interconnect interface. While this comes at the cost of a small area overhead, it allows for a plug-and-play usage for different implementations of a specific primitive. 


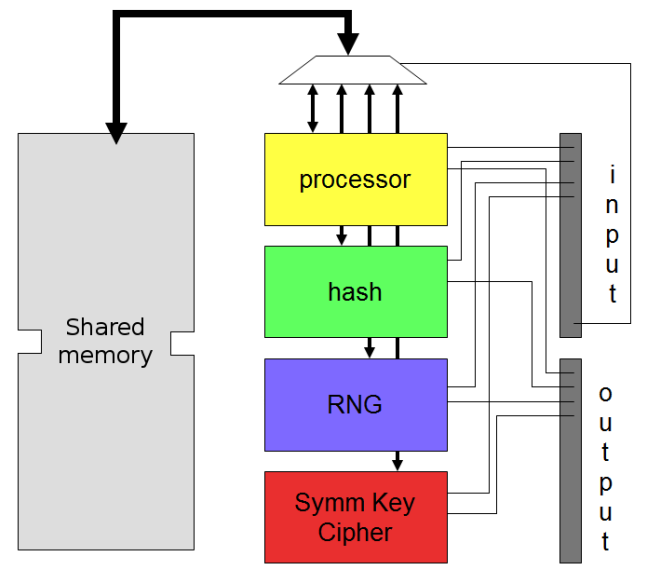

Fig. 4. Hardware architecture of cryptographic core

The complete cryptographic core is wrapped as an IP core component for Xilinx EDK. Together with a software driver, the IP component can easily be attached in any hardware design. This provides easy interaction with the communication core.

We now describe these four cryptographic primitives more into detail.

1) Elliptic curve coprocessor: The elliptic curve operations needed for point multiplication, signature generation and signature verification are: modular inversion, modular addition, modular subtraction, modular multiplication, elliptic curve point addition, and elliptic curve point multiplication. All these operations can be executed by means of an algorithm that only performs modular additions and modular multiplications. Since the total design is optimized towards area, only one instance of both mathematical functions is implemented. These two hardware implementations, with some register and control overhead, are the data path of a cryptographic coprocessor. This is shown in the middle rectangle in Fig. 5. The data memory for the data path is shown as the 'Work memory' in Fig. 5.

The control unit in the elliptic curve coprocessor fetches, decodes, and executes the required instructions. These instructions are stored in the instruction memory. A small 32-bit instruction interpreter is used to translate the algorithms for the elliptic curve processor (written in an assembly like language) into binary code. These algorithms are stored in the instruction memory. This memory consists of 3 programs: a signature generation program, a signature verification program and a program for elliptic point multiplications.

In the design of the elliptic curve processor, attention has been given to countermeasures against Simple Power Analysis (SPA) attacks [9]. This has been done by making the execution time of the algorithms independent of the processed keys. Countermeasures against Differential Power Analysis (DPA) and other forms of side-channel attacks have not yet been implemented.

Through the communication unit, input and output data can be written to or read from the work memory. For even more

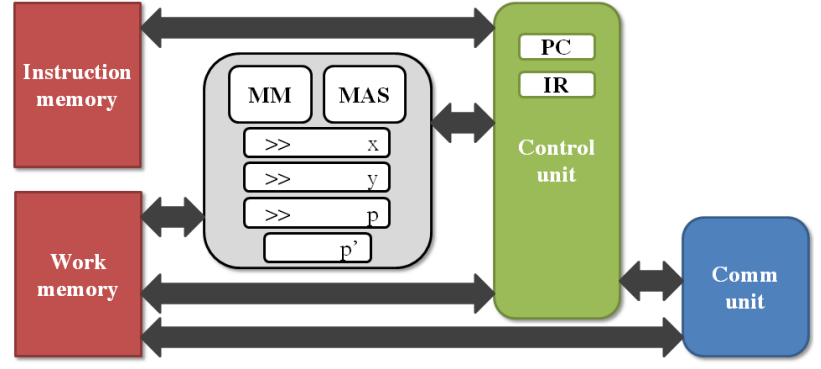

Fig. 5. Architecture of the elliptic curve coprocessor

detailed information regarding the coprocessor we suggest the reader to look in to [12].

2) Hash function: The hash function used in the STRES component is the SHA2-family member with a bit length of 256 bits. The implementation of the hash function is wrapped to connect it to the shared memory.

3) RNG component: The random number generator generates 256-bit random numbers. The randomness comes from the oscillator rings, as described in [13]. This component is also wrapped to connect to the shared memory.

4) Symmetric Key Cipher: For the symmetric key cipher a small implementation is made of a 128 bit AES component. This small implementation is based on the work done in [4], by Chodowiec and Gaj. The most beneficial factor in this implementation is that BRAM is used for the storage of the AES state.

To use a symmetric key cipher in a secure way a mode of operation is required. To reduce the overhead to a minimum while giving protection against chosen plaintext attacks the Cipher Block Chaining (CBC) mode is used. A drawback of using $\mathrm{CBC}$ is that there is error propagation. If, during transmission, an error occurs in a certain data block, all data blocks following this data block will be erroneously decrypted. When authenticating the bitstream shows there was an error, the bitstream is discarded. Hence, in this case, it does not matter whether there is fault propagation or not.

\section{TIMINGS AND SIZES}

It is obvious that the STRES component is overhead for the main application and is thus to be as small as possible. To give the reader a idea of how much overhead the STRES component takes, we summarized timing results and sizes of the two most important cores in this section.

\section{A. Communication core}

Since the efficiency of the communication core highly depends on the quality of the internet connection, it is useless to give exact timing results on transferring a bitstream to the FPGA. However, it is worth mentioning that writing the bitstream into the Flash, using a local private network, takes roughly half of the time compared by doing it by means of Xilinx Impact [16]. Xilinx Impact is the default software for writing bitstreams to an FPGA, which is included in the 
Xilinx Design Suite. Moreover, this approach is much less error prone.

The software for remote reconfiguration, including the communication (nIP) and the storage of a valid bitstream into Flash, requires less than 32 kBytes program memory of the MicroBlaze. The total program memory takes $46 \mathrm{kBytes}$. This includes the nIP, Flash write, and the drivers for the extra IP cores.

\section{B. Cryptographic core}

Although the cryptographic core is designed with flexibility in mind, only one set of results is given. The cryptographic processor can work on different word sizes. This allows for a flexible trade-off feature between the quantity of used reconfigurable fabric versus the throughput of the core. The results presented here are for a 32-bit word version of the coprocessor. Table I gives the results of every single cryptographic primitive, as discussed in Sec. V-B, and for the complete system. All these values are generated using the Xilinx development tools, release version 12.1, application version M53.d, targeting for a Xilinx Virtex 5 XC5VFX70T. Although several DSP slices are available in this device, none of these are used. The reason for this is that we want to give figures for the cryptographic core that are vendor independent.

TABLE I

TIMING RESULTS AND SIZES OF THE DIFFERENT PRIMITIVES IN THE STRES COMPONENT

\begin{tabular}{lrrr}
\hline Algorithm & Time $(\mu \mathrm{s})$ & \multicolumn{2}{c}{ Size } \\
& & slices & BRAM \\
\hline Elliptic curve signature generation & 58005.14 & 1646 & 10 \\
Elliptic curve signature verification & 207873.16 & & \\
SHA 256 & 4.91 & 599 & 0 \\
Random Number Generator & 0.47 & 334 & 0 \\
AES-128 Encryption & 30.56 & 392 & 10 \\
AES-128 Decryption & & 3871 & 21 \\
\hline crypto IP core & & 6882 & 72 \\
total project & & & \\
\hline
\end{tabular}

\section{CONCLUSION AND FUTURE WORK}

We have described a compact module, called STRES, for secure remote reconfiguration that can be easily added to an FPGA-based embedded system. It is based on the full STS security protocol to provide data confidentiality, mutual entity authentication, and mutual, explicit key authentication. It is the first complete system that implements in the same time communication by TCP/IP together with public key based security.

Future work will focus on two different aspects: partial reconfiguration and further improvements of the communication core. The partial reconfiguration could offer the possibility of leaving the static part of the FPGA online while only the dynamic part is reconfigured. Another option is that a part of the main application stays operational while the other part can be reconfigured. The other aspect for future work will try to reduce the communication unit.

\section{ACKNOWLEDGEMENT}

The STRES project has been supported financially by the IWT - Flemish Agency for Innovation by Science and Technology. Jo Vliegen was partially funded by the IOF funds of the KULeuven. The authors would like to thank the anonymous referees whose comments have greatly improved the paper.

\section{REFERENCES}

[1] M. Abdalla, M. Bellare, and P. Rogaway, The oracle Diffie-Hellman assumptions and an analysis of DHIES, Topics in Cryptology - CT-RSA 2001, 2001, pp. 143-158, Ed. D. Naccache, Lecture Notes in Computer Science, Vol. 2020, Springer - Berlin/Heidelberg.

[2] B. Badrignans, R. Elbaz, and L. Torres, Secure FPGA Configuration Architecture Preventing System Downgrade, Proceeding of the 18th International Conference on Field Programmable Logic and Applications, pp. 317-322, 2008

[3] M. Bellare, R. Canetti, and H. Krawczyk, Keying Hash Functions for Message Authentication, Proceedings of the 16th Annual International Cryptology Conference on Advances in Cryptology, 1996, pp. 1-15, Springer-Verlag London.

[4] P. Chodowiec, and K. Gaj, Very Compact FPGA Implementation of the AES Algorithm, Proceedings of the 5th international workshop on Cryptographic Hardware and Embedded Systems (CHES), 2003, pp. 319333, 2003, Eds. C. D. Walter and Ç. K. Koç and C. Paar, Lecture Notes in Computer Science, Springer.

[5] F. Devic, L. Torres, and B. Badrignans, Secure Protocol Implementation for Remote Bitstream Update Preventing Replay Attacks on FPGA, Proceeding of the 20th International Conference on Field Programmable Logic and Applications, pp. 179-182, 2010.

[6] S. Drimer, Security for volatile FPGAs, PHD thesis, University of Cambridge, Computer Laboratory (2009).

[7] A. Menezes, P. C. van Oorschot, and S. Vanstone, Handbook of Applied Cryptography, CRC Press, 1997.

[8] N. Koblitz, Elliptic curve cryptosystems, in Mathematics of Computation 48, 1987, pp. 203-209, 1987.

[9] P. Kocher, J. Jaffe, B. Jun, Differential Power Analysis, Proceedings of Advances in Cryptology - Crypto 99, Lecture Notes In Computer Science Vol. 1666, pp 388-397, 1999.

[10] V. Miller, Use of elliptic curves in cryptography, Advances in cryptology CRYPTO 85, 1985, pp. 417-426, Lecture notes in computer sciences 218 , Springer-Verlag New York.

[11] Recommended Elliptic Curves for Federal Government Use, July 1999, http://csrc.nist.gov/groups/ST/toolkit/documents/dss/NISTReCur.pdf, retrieved April 6, 2011.

[12] J. Vliegen, N. Mentens, J. Genoe, A. Braeken, S. Kubera, A. Touhafi, I. Verbauwhede, A compact FPGA-based architecture for elliptic curve cryptography over prime fields, Proceedings of 21st IEEE International Conference on Application-specific Systems 2010, pp. 313-316, 2010.

[13] K. Wold, and C. H. Tan, Analysis and enhancement of random number generator in FPGA based on oscillator rings, International Journal Reconfigurational Computing, 2009, pp. 4:1-4:8, Hindawi Publishing Corp. New York.

[14] uIP, April 2011, http://www.sics.se/ adam/uip/index.php/Main_Page, retrieved April 15, 2011

[15] Leight weight IP, May 2004, http://www.sics.se/ãdam/lwip/, retrieved April 15, 2011.

[16] Xilinx Design Suite, 2011, http://www.xilinx.com/products/designtools/ise-design-suite/index.htm, retrieved April 22, 2011.

[17] F. Devic, L. Torres, B. Badrignans, Secure Protocol Implementation for Remote Bitstream Update Preventing Replay Attacks on FPGA, Proceeding of the 20th International Conference on Field Programmable Logic and Applications, pp. 179-182, 2010. 